\title{
Some Numerical Methods and Comparisons for Solving Mathematical Model of Surface Decontamination by Disinfectant Solution
}

\author{
${ }^{1}$ Chai Jin Sian, ${ }^{2}$ Yeak Su Hoe* and ${ }^{3}$ Ali H. M. Murid \\ $\mathbf{1 , 2 , 3}$ Department of Mathematical Sciences, Faculty of Science \\ Universiti Teknologi Malaysia, 81310 Johor Bahru, Malaysia \\ ${ }^{3}$ UTM Centre for Industrial and Applied Mathematics \\ Ibnu Sina Institute for Scientific and Industrial Research \\ Universiti Teknologi Malaysia, 81310 Johor Bahru, Malaysia \\ ${ }^{*}$ Corresponding author: s.h.yeak@utm.my
}

Article history

Received: 7 February 2018

Received in revised form: No revision

Accepted: 11 February 2018

Published on line: 1 December 2018

\begin{abstract}
A mathematical model is considered to determine the effectiveness of disinfectant solution for surface decontamination. The decontamination process involved the diffusion of bacteria into disinfectant solution and the reaction of the disinfectant killing effect. The mathematical model is a reaction-diffusion type. Finite difference method and method of lines with fourth-order Runge-Kutta method are utilized to solve the model numerically. To obtain stable solutions, von Neumann stability analysis is employed to evaluate the stability of finite difference method. For stiff problem, Dormand-Prince method is applied as the estimated error of fourth-order Runge-Kutta method. MATLAB programming is selected for the computation of numerical solutions. From the results obtained, fourth-order Runge-Kutta method has a larger stability region and better accuracy of solutions compared to finite difference method when solving the disinfectant solution model. Moreover, a numerical simulation is carried out to investigate the effect of different thickness of disinfectant solution on bacteria reduction. Results show that thick disinfectant solution is able to reduce the dimensionless bacteria concentration more effectively.
\end{abstract}

Keywords Reaction-diffusion; finite difference method; method of lines; fourth-order Runge-Kutta method; von Neumann stability analysis; Dormand-Prince method.

Mathematics Subject Classification 65M06, 65M12, 65M20

\section{Introduction}

Natural products like disinfectants show healing and antibacterial properties which make them very helpful in the field of biology. In the past, disinfectants were used by people to treat various illnesses and heal wound. Apart from that, French green disinfectants had been employed to 
cure Buruli ulcer, a chronic and necrotizing skin disease caused by Mycobacterium ulcerans [1]. Hence, disinfectant have the ability to kill many kinds of pathogenic bacteria by buffering the aqueous $\mathrm{pH}$ and oxidation state during the antibacterial process $[2,3,4]$.

A lot of researches had been done regarding the process of decontamination to provide a clean and healthy environment. For examples, the surface decontamination by clay solution, the problem of aquifer decontamination by pumping [6], the cleaning of sea pollution using pumping process carried on by a ship [7] and the electrokinetic remediation of nuclear wastecontaminated soils [8]. They used parabolic partial differential equations (PDEs) to formulate their mathematical models. The models were then solved using mathematical methods such as Laplace transform method, finite difference method, finite volume method or finite element method in order to acquire the approximate solutions.

In [5], an analytical solution has been found for the clay solution modelat one fixed point, $\hat{x}=0$ by using Laplace transform method. However, the complexity of the mathematical model leads to difficulties in obtaining an analytical solution for the range of finite domain, $0 \leq \hat{x} \leq h$. Consequently, numerical methods are more preferable to be implemented in the disinfectant solution model to provide an approximation to the exact solution for the case $0 \leq \hat{x} \leq h$. This is because numerical methods are applicable to solve various types of mathematical models. We note that the model in [5] is also applicable to not just clay solution, but also to other disinfectant solution.

In this paper, finite difference method (FDM) and method of lines (MOL) with fourthorder Runge-Kutta method (RK4) are applied to solve the disinfectant solution model in [5] numerically. To ensure that the numerical solutions obtained are stable and high in accuracy, von Neumann stability analysis is employed to evaluate the stability of FDM. For stiff problem, Dormand-Prince (DOPRI) method is adopted as the estimated error of RK4 for solving the model. Furthermore, comparisons are made between FDM and RK4 about the stability and accuracy of solutions generated. Apart from that, different thickness of disinfectant solution is applied in the numerical simulation of disinfectant solution model so that the effect of the thickness of disinfectant solution on bacteria reduction can be identified. In this research, MATLAB programming has been developed to execute numerical solutions for the model.

\section{Methodology}

In this section, the mathematical formulation of the disinfectant solution model as well as the application of FDM and MOL with RK4 in the model will be presented. Other than that, the stability analysis of FDM and RK4 will be conducted by using von Neumann stability analysis and DOPRI method, respectively.

\subsection{Mathematical Modelling of Surface Decontamination by Disinfectant Solution}

Based on [5], there are several assumptions that need to be considered for the mathematical modelling on the effectiveness of disinfectant solution for surface decontamination. Firstly, the bacteria are assumed to diffuse into the disinfectant solution and then eliminated by the disinfectant killing effect. Secondly, the bottom of contaminated surface is assumed to be hard and impermeable. The interaction process of disinfectant solution and contaminant is demonstrated in Figure 1. 


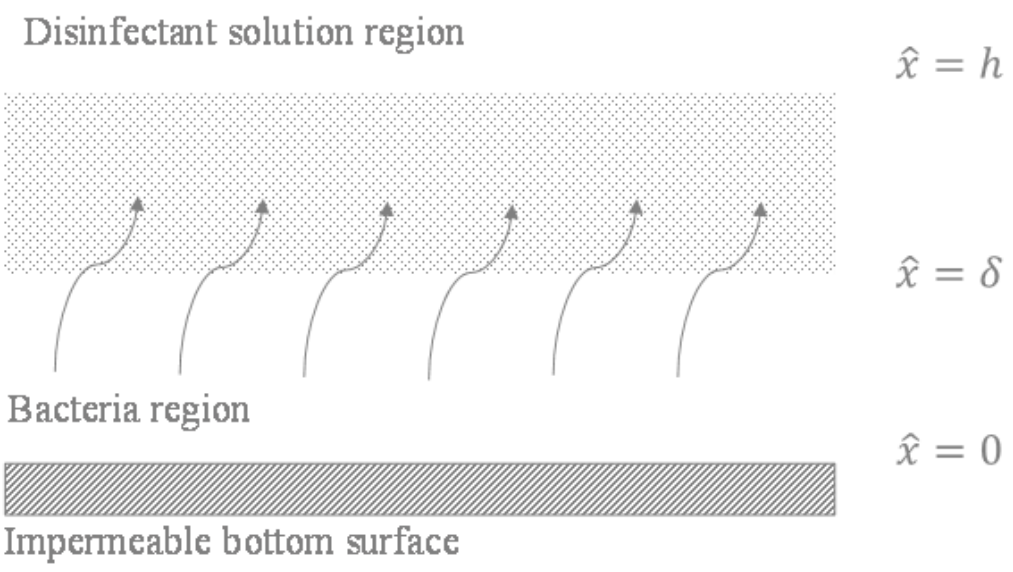

Figure 1: Physical Condition of Interaction Process between Disinfectant Solution and Contaminant

Bacteria concentration is closely related to the time taken, $\hat{t}$ and the thickness of region, $\hat{x}$. As time passes, the bacteria move upward from a lower region to a higher region. When the bacteria enter disinfectant solution region, they are killed by the disinfectant killing effect. Hence, this paper considers the bacteria movement invertical direction only as one dimensional problem. The movement of bacteria against time is presented in Figure 2.

\section{Thickness of region}

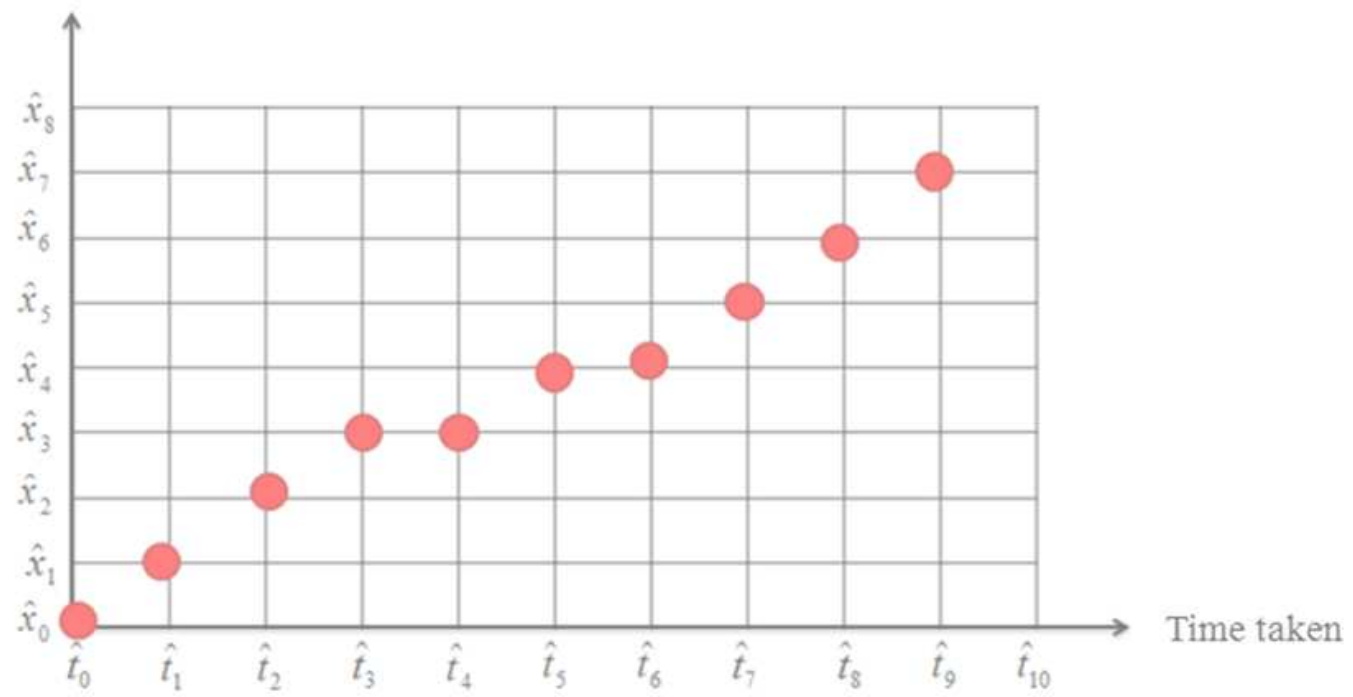

Figure 2: Cartesian Coordinate of the Movement of Bacteria against Time

The variables and parameters involved for the formulation of the disinfectant solution model are shown as follows: 


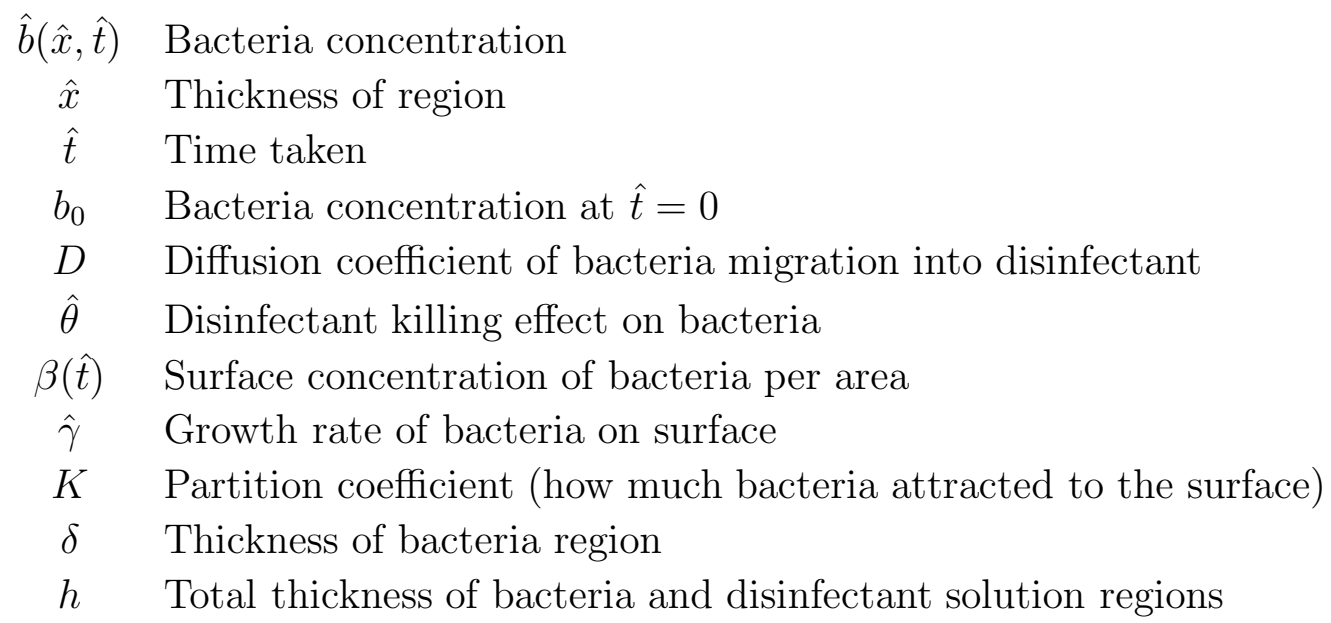

The mathematical model of surface decontamination by disinfectant solution is formulated as follows [5]:

Model A

$$
\frac{\partial \hat{b}}{\partial \hat{t}}=D \frac{\partial^{2} \hat{b}}{\partial \hat{x}^{2}}-\hat{\theta} \hat{b}, \quad 0<\hat{x}<h, \hat{t}>0
$$

Boundary conditions:

$$
\begin{array}{lll}
\hat{x}=0: & \left.K \delta \frac{\partial \hat{b}}{\partial \hat{t}}\right|_{\hat{x}=0}=\left.D \frac{\partial \hat{b}}{\partial \hat{x}}\right|_{\hat{x}=0}+\hat{\gamma} K \delta \hat{b}(0, \hat{t}), & \hat{t}>0 \\
\hat{x}=h: & \hat{b}(h, \hat{t})=0 \text { or }\left.\frac{\partial \hat{b}}{\partial \hat{x}}\right|_{\hat{x}=h}=0, & \hat{t}>0
\end{array}
$$

Initial condition:

$$
\hat{b}(\hat{x}, 0)= \begin{cases}b_{0}, & 0 \leq \hat{x} \leq \delta \\ 0, & \delta<\hat{x} \leq h\end{cases}
$$

To facilitate mathematical analysis, Model A needs to be transformed into a dimensionless form. Let the dimensionless variables and parameters be

$$
\tilde{b}=\frac{\hat{b}}{b_{0}}, \quad \tilde{x}=\frac{\hat{x}}{h}, \quad \tilde{t}=\frac{D \hat{t}}{h^{2}}, \quad \theta=\frac{h^{2} \hat{\theta}}{D}, \quad \gamma=\frac{(h \delta) \hat{\gamma}}{D} .
$$

Then, substitute all these dimensionless terms into Model A to form Model B [5].

$\underline{\text { Model B }}$ (Non dimensionalization of Model A)

$$
\frac{\partial \tilde{b}}{\partial \tilde{t}}=\frac{\partial^{2} \tilde{b}}{\partial \tilde{x}^{2}}-\theta \tilde{b}, \quad 0<\tilde{x}<1, \tilde{t}>0
$$




\section{Boundary conditions:}

$$
\begin{array}{lll}
\tilde{x}=0: & \left.\frac{K \delta}{h} \frac{\partial \tilde{b}}{\partial \tilde{t}}\right|_{\tilde{x}=0}=\left.\frac{\partial \tilde{b}}{\partial \tilde{x}}\right|_{\tilde{x}=0}+\gamma K \tilde{b}(0, \tilde{t}), & \tilde{t}>0 \\
\tilde{x}=1: & \tilde{b}(1, \tilde{t})=0, \text { or }\left.\frac{\partial \tilde{b}}{\partial \tilde{x}}\right|_{\tilde{x}=1}=0, & \tilde{t}>0
\end{array}
$$

Initial condition:

$$
\tilde{b}(\tilde{x}, 0)= \begin{cases}1, & 0 \leq \tilde{x} \leq \frac{\delta}{h} \\ 0, & \frac{\delta}{h}<\tilde{x} \leq 1\end{cases}
$$

To remove $\theta$ in equation (5), assume the variables used in Model B to be

$$
\tilde{b}(\tilde{x}, \tilde{t})=b(x, t), \quad \tilde{x}=\frac{x}{\sqrt{\theta}}, \quad \tilde{t}=\frac{t}{\theta} .
$$

After that, substitute all these terms into Model B to yield Model C [5].

Model C (Modified Model B)

$$
\frac{\partial b}{\partial t}=\frac{\partial^{2} b}{\partial x^{2}}-b, \quad 0<x<\sqrt{\theta}, t>0
$$

Boundary conditions:

$$
\begin{array}{lll}
x=0: & \left.\tau \frac{\partial b}{\partial t}\right|_{x=0}=\left.\frac{\partial b}{\partial x}\right|_{x=0}+\lambda b(0, t), & t>0 \\
x=\sqrt{\theta}: & b(\sqrt{\theta}, t)=0 \text { or }\left.\frac{\partial b}{\partial x}\right|_{x=\sqrt{\theta}}=0, & t>0
\end{array}
$$

\section{Initial condition:}

$$
b(x, 0)= \begin{cases}1, & 0 \leq x \leq \frac{\delta \sqrt{\theta}}{h} \\ 0, & \frac{\delta \sqrt{\theta}}{h}<x \leq \sqrt{\theta}\end{cases}
$$

where $\tau=K \delta \sqrt{\theta} / h$ and $\lambda=\gamma K / \sqrt{\theta}$ are positive constants.

In [5], Model C is further simplified by extending the interval $0<x<\sqrt{\theta}$ to infinity and then solved by using Laplace transform. In this paper, Model C is utilized as the disinfectant solution model to determine the effectiveness of disinfectant solution for surface decontamination.

\subsection{Implementation of Numerical Methods along with Stability Analysis}

In this section, the implementation of FDM and MOL with RK4 in the disinfectant solution model along with their stability analysis will be explained in details. 


\subsubsection{FDM in Disinfectant Solution Model}

First of all, discretise the independent variables $x$ and $t$ into grid points as follows:

$$
\left(x_{j}, t_{n}\right)=[(j-1) \cdot \Delta x,(n-1) \cdot \Delta t], \quad 1 \leq j \leq N, 1 \leq n \leq M,
$$

where the $x$ step size, $\Delta x=\sqrt{\theta} / N-1$ and $\Delta t$ denotes the $t$ step size, while $N$ and $M$ are the total number of nodes for variables $x$ and $t$, respectively. After discretisation, the function $b(x, t)$ can be written as

$$
b_{j}^{n}=b\left(x_{j}, t_{n}\right) .
$$

Then, substitute the first order forward difference formula for time derivative and also first and second order central difference formulas for spatial derivatives involved in the disinfectant solution model to yield the following equations:

$$
b_{j}^{n+1}=b_{j}^{n}+\frac{\Delta t}{(\Delta x)^{2}}\left(b_{j-1}^{n}-2 b_{j}^{n}+b_{j+1}^{n}\right)-\Delta t b_{j}^{n}+O\left[\Delta t,(\Delta x)^{2}\right], \quad 1<j<N, 1 \leq n \leq M-1
$$

\section{Boundary conditions:}

$$
\begin{array}{ll}
b_{1}^{n+1}=-\frac{\Delta t}{2 \tau \Delta x} b_{0}^{n}+\left(1+\frac{\Delta t}{\tau} \lambda\right) b_{1}^{n}+\frac{\Delta t}{2 \tau \Delta x} b_{2}^{n}+O\left[\Delta t,(\Delta x)^{2}\right], & 1 \leq n \leq M-1 \\
b_{N}^{n}=0, & 1<n \leq M-1
\end{array}
$$

Initial condition:

$$
b_{j}^{1}=\left\{\begin{array}{ll}
1, & 0 \leq x_{j} \leq \frac{\delta \sqrt{\theta}}{h} \\
0, & \frac{\delta \sqrt{\theta}}{h}<x_{j} \leq \sqrt{\theta}
\end{array}, \quad 1 \leq j \leq N\right.
$$

where $b_{0}^{n}=\left[-2 \tau b_{2}^{n}+4 \tau b_{1}^{n}+\Delta x b_{2}^{n}+2 \tau \cdot(\Delta x)^{2} b_{1}^{n}+2 \lambda \cdot(\Delta x)^{2} b_{1}^{n}\right] /(\Delta x+2 \tau)$.

By applying FDM, the disinfectant solution model can be solved explicitly by considering the initial and boundary conditions provided. Although FDM is easy to be adopted to approximate various kinds of PDEs but it does not guarantee that stable numerical solutions can be obtained. Hence, it is necessary to evaluate the stability of FDM by employing the von Neumann stability analysis. The application of von Neumann stability analysis in the disinfectant solution model can be shown as follows:

1. According to equation (13), the reaction-diffusion equation of disinfectant solution model is presented as

$$
b_{j}^{n+1}=b_{j}^{n}+\frac{\Delta t}{(\Delta x)^{2}}\left(b_{j-1}^{n}-2 b_{j}^{n}+b_{j+1}^{n}\right)-\Delta t b_{j}^{n}+O\left[\Delta t,(\Delta x)^{2}\right] .
$$

2. Assume the Fourier term expression for function $b\left(x_{j}, t_{n}\right)$ as

$$
b\left(x_{j}, t_{n}\right)=\sum_{k=-\infty}^{\infty} B_{k}\left(t_{n}\right) \phi_{k}\left(x_{j}\right)=\sum_{k=-\infty}^{\infty} B_{k}^{n} e^{i 2 \pi k j \Delta x} .
$$


By letting $\theta=2 \pi k \Delta x$, equation (17) becomes

$$
b\left(x_{j}, t_{n}\right)=\sum_{\theta=-\infty}^{\infty} B_{\theta}^{n} \phi_{\theta j}=\sum_{\theta=-\infty}^{\infty} B_{\theta}^{n} e^{i j \theta}
$$

3. Drop the truncation errors, $O\left[\Delta t,(\Delta x)^{2}\right]$ in equation (13) and then substitute equation (18) into the reaction-diffusion equation to produce

$$
\begin{aligned}
& \sum_{\theta=-\infty}^{\infty} B_{\theta}^{n+1} e^{i j \theta} \\
& =\sum_{\theta=-\infty}^{\infty} B_{\theta}^{n} e^{i j \theta}+\frac{\Delta t}{(\Delta x)^{2}} \sum_{\theta=-\infty}^{\infty}\left[B_{\theta}^{n} e^{i(j-1) \theta}-2 B_{\theta}^{n} e^{i j \theta}+B_{\theta}^{n} e^{i(j+1) \theta}\right]-\Delta t \sum_{\theta=-\infty}^{\infty} B_{\theta}^{n} e^{i j \theta} .
\end{aligned}
$$

4. Divide equation (19) with $\sum B_{\theta}^{n} e^{i j \theta}$ to obtain

$$
\frac{B_{\theta}^{n+1}}{B_{\theta}^{n}}=1+\frac{\Delta t}{(\Delta x)^{2}}\left[e^{-i \theta}-2+e^{i \theta}\right]-\Delta t \text { or } \frac{B_{\theta}^{n+1}}{B_{\theta}^{n}}=1-4 \frac{\Delta t}{(\Delta x)^{2}} \sin ^{2}\left(\frac{\theta}{2}\right)-\Delta t .
$$

5. For stability,

$$
\left|\frac{B_{\theta}^{n+1}}{B_{\theta}^{n}}\right|<1 \text { or }\left|1-4 \frac{\Delta t}{(\Delta x)^{2}} \sin ^{2}\left(\frac{\theta}{2}\right)-\Delta t\right|<1
$$

6. Hence, the range for the value of $\Delta t$ is

$$
0<\Delta t<\frac{2}{\frac{4}{(\Delta x)^{2}} \sin ^{2}\left(\frac{\theta}{2}\right)+1}
$$

7. To ensure that the numerical solutions are bounded, we specify

$$
0<\Delta t<\frac{2}{\frac{4}{(\Delta x)^{2}}+1}
$$

\subsubsection{MOL with RK4 in Disinfectant Solution Model}

MOL is a special FDM which transforms a given PDE into a system of ordinary differential equations (ODEs). It works by replacing the spatial derivatives in PDE with algebraic approximations [9]. To implement MOL in the disinfectant solution model, the first step is to discretise the independent variable $x$ into grid points,

$$
x_{j}=(j-1) \cdot \Delta x, \quad 1 \leq j \leq N
$$

After discretisation, the function $b(x, t)$ can be written as

$$
b_{j}(t)=b\left(x_{j}, t\right) .
$$


Next, substitute second and first order central difference formulas for the spatial derivatives in equations (9) and (10) to get

$$
\frac{d}{d t} b_{j}(t)=\frac{1}{(\Delta x)^{2}}\left[b_{j-1}(t)-2 b_{j}(t)+b_{j+1}(t)\right]-b_{j}(t)+O\left[(\Delta x)^{2}\right], \quad 1<j<N
$$

and

$$
\frac{d}{d t} b_{1}(t)=\frac{1}{2 \tau \Delta x}\left[b_{2}(t)-b_{0}(t)\right]+\frac{\lambda}{\tau} b_{1}(t)+O\left[(\Delta x)^{2}\right],
$$

where

$$
b_{0}(t)=\frac{\left[2 \lambda(\Delta x)^{2}+2 \tau(\Delta x)^{2}+4 \tau\right] b_{1}(t)+(\Delta x-2 \tau) b_{2}(t)}{2 \tau+\Delta x} .
$$

Equation (23) can be rewritten as

$$
\frac{d}{d t} b_{1}(t)=\left[\frac{2 \lambda \Delta x-(\Delta x)^{2}-2}{\Delta x(2 \tau+\Delta x)}\right] b_{1}(t)+\frac{2}{\Delta x(2 \tau+\Delta x)} b_{2}(t)+O\left[(\Delta x)^{2}\right]
$$

by substituting the value of $b_{0}(t)$ into equation (23). Finally, the disinfectant solution model can be presented as a system of ODEs as follows:

$$
\frac{d}{d t}\left[\begin{array}{c}
b_{1}(t) \\
b_{2}(t) \\
\vdots \\
b_{N-2}(t) \\
b_{N-1}(t)
\end{array}\right]=\left[\begin{array}{c}
{\left[\frac{2 \lambda \Delta x-(\Delta x)^{2}-2}{\Delta x(2 \tau+\Delta x)}\right] b_{1}(t)+\frac{2}{\Delta x(2 \tau+\Delta x)} b_{2}(t)} \\
\frac{1}{(\Delta x)^{2}}\left[b_{1}(t)-2 b_{2}(t)+b_{3}(t)\right]-b_{2}(t) \\
\vdots \\
\frac{1}{(\Delta x)^{2}}\left[b_{N-3}(t)-2 b_{N-2}(t)+b_{N-1}(t)\right]-b_{N-2}(t) \\
\frac{1}{(\Delta x)^{2}}\left[b_{N-2}(t)-2 b_{N-1}(t)+b_{N}(t)\right]-b_{N-1}(t)
\end{array}\right]+O\left[(\Delta x)^{2}\right]
$$

According to equation (11), when $x=\sqrt{\theta}, b_{N}(t)=0$. This means that there are no more bacteria after a cut-off distance. So, the system of ODEs becomes

$$
\frac{d}{d t}\left[\begin{array}{c}
b_{1}(t) \\
b_{2}(t) \\
\vdots \\
b_{N-2}(t) \\
b_{N-1}(t)
\end{array}\right]=\left[\begin{array}{c}
{\left[\frac{2 \lambda \Delta x-(\Delta x)^{2}-2}{\Delta x(2 \tau+\Delta x)}\right] b_{1}(t)+\frac{2}{\Delta x(2 \tau+\Delta x)} b_{2}(t)} \\
\frac{1}{(\Delta x)^{2}}\left[b_{1}(t)-2 b_{2}(t)+b_{3}(t)\right]-b_{2}(t) \\
\vdots \\
\frac{1}{(\Delta x)^{2}}\left[b_{N-3}(t)-2 b_{N-2}(t)+b_{N-1}(t)\right]-b_{N-2}(t) \\
\frac{1}{(\Delta x)^{2}}\left[b_{N-2}(t)-2 b_{N-1}(t)\right]-b_{N-1}(t)
\end{array}\right]+O\left[(\Delta x)^{2}\right] .
$$

The initial equation in equation (12) can be expressed as

$$
b_{j}(t)= \begin{cases}1, & 0 \leq x_{j} \leq \delta \sqrt{\theta} / h \\ 0, & \delta \sqrt{\theta} / h \leq x_{j} \leq \sqrt{\theta}\end{cases}
$$


or in matrix form as

$$
\left[\begin{array}{c}
b_{1}(0) \\
\vdots \\
b_{r}(0) \\
b_{r+1}(0) \\
\vdots \\
b_{N}(0)
\end{array}\right]=\left[\begin{array}{c}
1 \\
\vdots \\
1 \\
0 \\
\vdots \\
0
\end{array}\right], \quad 1<r<N .
$$

Having completed the implementation of MOL in the disinfectant solution model, the next step is finding the solutions of Initial Value Problems (IVPs) in system of ODEs by employing RK4. RK4 is the most popular single-step numerical method for solving IVPs because it can generate high accuracy results when compared with the exact solutions and its accuracy can be further improved by reducing the step size used [10]. The application of RK4 in the ODEs system can be shown as follows:

1. Firstly, discretise the independent variable, $t$ in system of ODEs into grid points as

$$
t_{n}=(n-1) \Delta t, \quad 1 \leq n \leq M .
$$

2. Then, assume the first order derivatives of function $b_{j}\left(t_{n}\right)$ for $1 \leq j \leq N-1$ to be

$$
\begin{aligned}
& \frac{d b_{1}}{d t_{n}}=f_{1}\left[t_{n}, b_{1}\left(t_{n}\right), b_{2}\left(t_{n}\right), \ldots, b_{N-1}\left(t_{n}\right)\right], \\
& \frac{d b_{2}}{d t_{n}}=f_{2}\left[t_{n}, b_{1}\left(t_{n}\right), b_{2}\left(t_{n}\right), \ldots, b_{N-1}\left(t_{n}\right)\right], \\
& \vdots \\
& \frac{d b_{N-1}}{d t_{n}}=f_{N-1}\left[t_{n}, b_{1}\left(t_{n}\right), b_{2}\left(t_{n}\right), \ldots, b_{N-1}\left(t_{n}\right)\right] .
\end{aligned}
$$

3. Next, introduce the vectors

$$
\begin{aligned}
& \mathbf{b}_{n}=\left[b_{1}\left(t_{n}\right), b_{2}\left(t_{n}\right), \ldots, b_{N-1}\left(t_{n}\right)\right]^{T}, \\
& \mathbf{f}\left(t_{n}, \mathbf{b}_{n}\right)=\left[f_{1}\left(t_{n}, \mathbf{b}_{n}\right), f_{2}\left(t_{n}, \mathbf{b}_{n}\right), \ldots, f_{N-1}\left(t_{n}, \mathbf{b}_{n}\right)\right]^{T}, \\
& \mathbf{K}_{i}=\left[k_{i, 1}, k_{i, 2}, \ldots, k_{i, N-1}\right]^{T}, i=1, \cdots, 4
\end{aligned}
$$

where

$$
\begin{aligned}
& \mathbf{K}_{1}=\Delta t \cdot \mathbf{f}\left(t_{n}, \mathbf{b}_{n}\right) \\
& \mathbf{K}_{2}=\Delta t \cdot \mathbf{f}\left(t_{n}+\frac{\Delta t}{2}, \mathbf{b}_{n}+\frac{\mathbf{K}_{1}}{2}\right), \\
& \mathbf{K}_{3}=\Delta t \cdot \mathbf{f}\left(t_{n}+\frac{\Delta t}{2}, \mathbf{b}_{n}+\frac{\mathbf{K}_{2}}{2}\right) \\
& \mathbf{K}_{4}=\Delta t \cdot \mathbf{f}\left(t_{n}+\Delta t, \mathbf{b}_{n}+\mathbf{K}_{3}\right) .
\end{aligned}
$$

4. Finally, RK4 formula for the disinfectant solution model can be stated in vector form as

$$
\mathbf{b}_{n+1}=\mathbf{b}_{n}+\frac{1}{6}\left(\mathbf{K}_{1}+2 \mathbf{K}_{2}+2 \mathbf{K}_{3}+\mathbf{K}_{4}\right)+O\left[(\Delta t)^{5},(\Delta x)^{2}\right], \quad n=1,2,3, \ldots, M-1 .
$$


Estimated error of RK4 is important to ensure that the results produced are stable and high in accuracy. Normally, the system of ODEs becomes numerically unstable when there exist stiff differential equations. Stiffness can be defined in terms of the eigenvalues of the Jacobian as follows [11] where the differentiation of $\mathbf{f}(t, \mathbf{b})$ is equal to matrix $A$.

Definition: A linear system $\mathbf{y}^{\prime}=A \mathbf{y}+\varphi(t)$ is said to be stiff if

1. Eigenvalues of matrix $A, \operatorname{Re} \lambda_{w}<0, w=1,2, \ldots, W$.

2. Stiffness ratio, $S \equiv \max _{w=1, \ldots, W}\left|\operatorname{Re} \lambda_{w}\right| / \min _{w=1, \ldots, W}\left|\operatorname{Re} \lambda_{w}\right|>>1$.

The system of ODEs of the disinfectant solution model stated in equation (26) can be presented in matrix form as follows:

$$
\begin{gathered}
=\left[\begin{array}{c}
b_{1}(t) \\
b_{2}(t) \\
b_{3}(t) \\
\vdots \\
b_{N-3}(t) \\
b_{N-2}(t) \\
b_{N-1}(t)
\end{array}\right] \\
=\left[\begin{array}{ccccccc}
\frac{2 \lambda \Delta x-(\Delta x)^{2}-2}{\Delta x(2 \tau+\Delta x)} & \frac{2}{\Delta x(2 \tau+\Delta x)} & 0 & \cdots & 0 & 0 & 0 \\
\frac{1}{(\Delta x)^{2}} & \frac{-2}{(\Delta x)^{2}}-1 & \frac{1}{(\Delta x)^{2}} & \cdots & 0 & 0 & 0 \\
0 & \frac{1}{(\Delta x)^{2}} & \frac{-2}{(\Delta x)^{2}}-1 & \cdots & 0 & 0 & 0 \\
\vdots & \ddots & \ddots & \ddots & \ddots & \ddots & \vdots \\
0 & 0 & 0 & \cdots & \frac{-2}{(\Delta x)^{2}}-1 & \frac{1}{(\Delta x)^{2}} & 0 \\
0 & 0 & 0 & \cdots & \frac{1}{(\Delta x)^{2}} & \frac{-2}{(\Delta x)^{2}}-1 & \frac{1}{(\Delta x)^{2}} \\
b^{2} \\
b_{N-2}(t) \\
b_{N-1}(t)
\end{array}\right]
\end{gathered}
$$

where matrix $A$ is

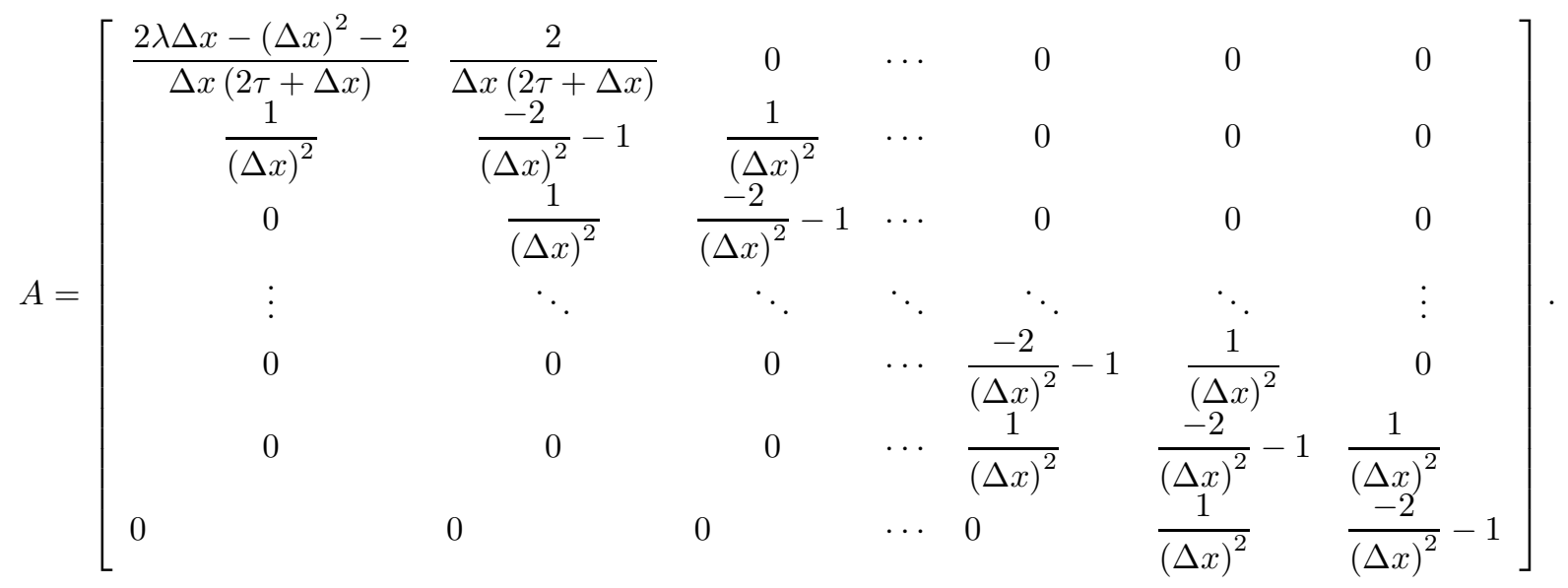

Then, the eigenvalues of matrix $A, \lambda_{w}$ can be acquired by solving the determinant

$$
\operatorname{det}\left(A-\lambda_{w} I\right)=0, \quad w=1,2, \ldots, N-1,
$$


where $I$ is an $(N-1) \times(N-1)$ identity matrix.

If the ODEs system is stiff, then an Adaptive Runge-Kutta method, DOPRI method will be applied as the estimated error of RK4 to produce stable and high accuracy solutions. The formula for DOPRI method can be represented by a Butcher Table as illustrated in Figure 3. For this research, an initial value for $\Delta t$ will be assigned for the DOPRI method to start the numerical computations. If the error is high, then the step size will be halved. Contrarily, if the error is very low, then the step size will be doubled. This procedure is repeated until the error produced meets the tolerance value that fixed on it. Eventually, stable and high accuracy results can be obtained with minimum computational effort.

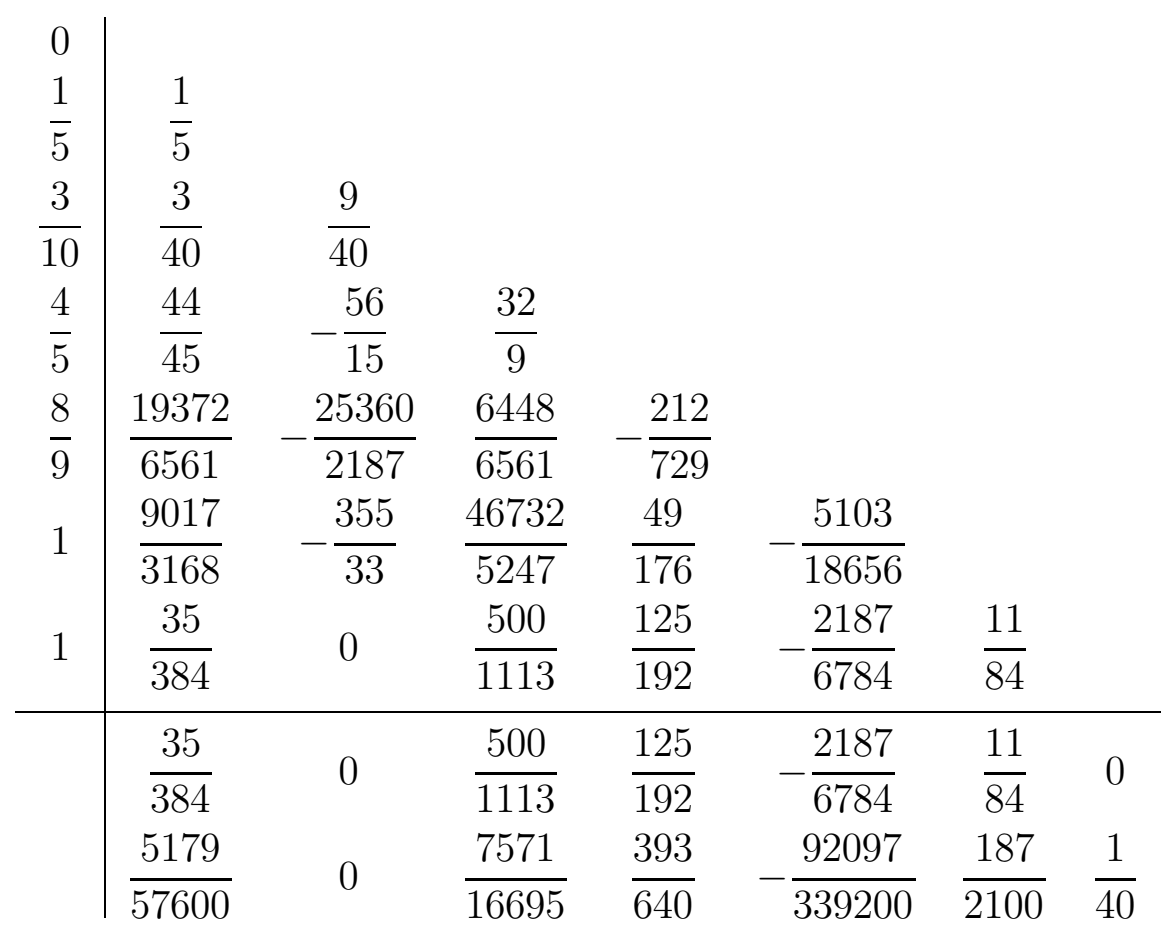

Figure 3: Butcher Table for DOPRI Method

\section{Results and Discussion}

In this section, FDM and MOL with RK4 will be utilized to solve the disinfectant solution model numerically. Besides that, the effect of the thickness of disinfectant solution on bacteria reduction will be investigated by applying different thickness of disinfectant solution in the numerical simulation of the disinfectant solution model.

\subsection{Parameter and Variable Setting}

Consider $\delta=0.000195 \mathrm{~m}$ and $h=0.0005 \mathrm{~m}$. While the other dimensionless parameters and variables values follow the following assumptions:

1. $\gamma=0.00001$

2. $K=0.5$ 
3. $\theta=36$

4. $0 \leq x \leq 6$

5. $0 \leq t \leq 1$

\subsection{Stability Analysis}

The stability regions of FDM versus RK4 when solving the disinfectant solution model are illustrated in Figure 4. Observe that RK4 has a larger stability region compared to FDM. This means that RK4 can perform the numerical simulation using larger time step size and this reduces the computational effort to execute stable numerical solutions.

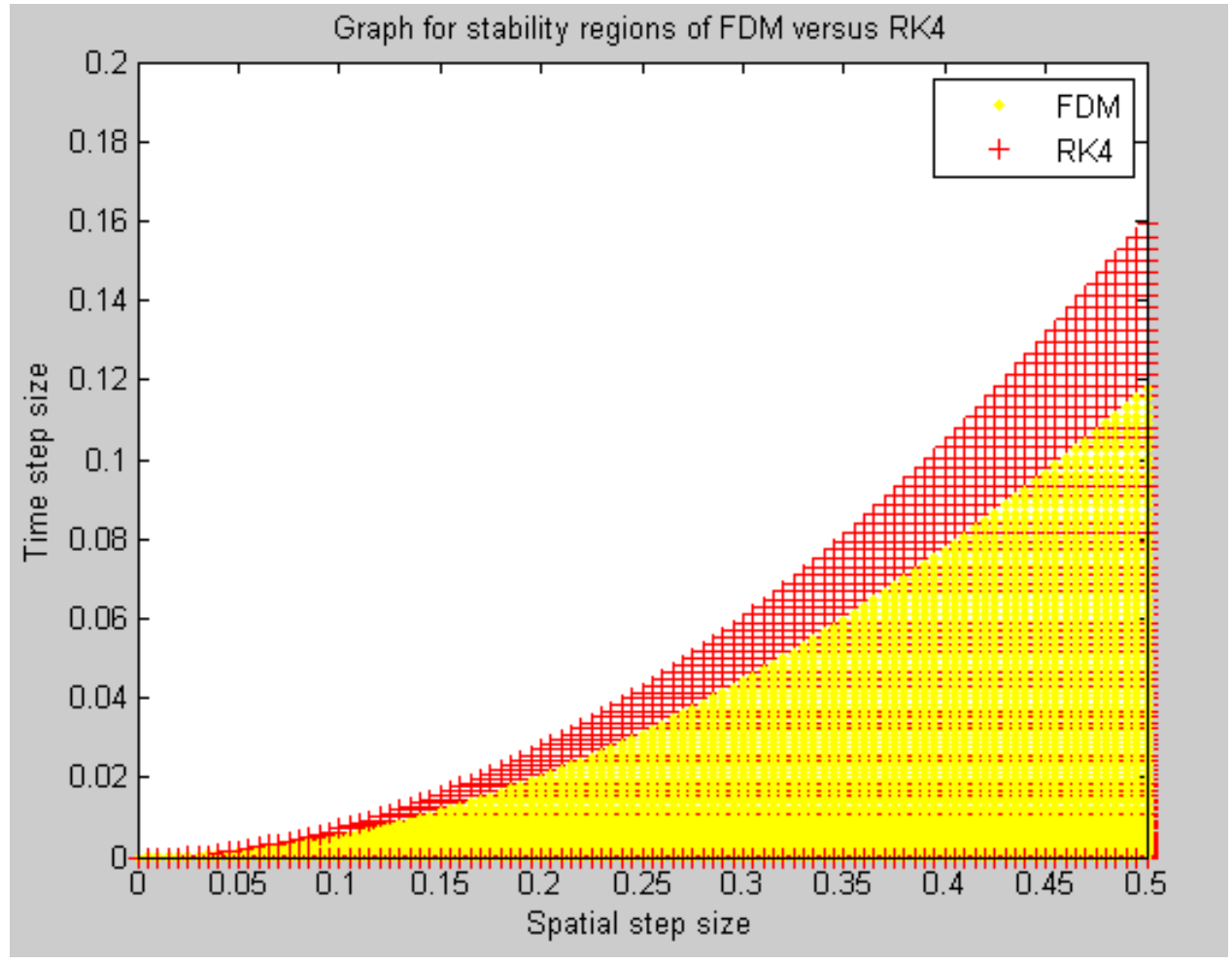

Figure 4: Stability Regions of FDM versus RK4

Apart from that, the stiffness of the ODEs system of disinfectant solution model can be determined by setting the spatial step size, $\Delta x=0.3$ into equation (29) in order to acquire all the eigenvalues for matrix $A$ as recorded in Table 1.

Note that all the eigenvalues of matrix $A$ in Table 1 are less than zero and the stiffness ratio,

$$
S=\frac{\max _{w=1, \ldots, 20}\left|\operatorname{Re} \lambda_{w}\right|}{\min _{w=1, \ldots, 20}\left|\operatorname{Re} \lambda_{w}\right|}=\frac{45.172559}{0.566139}=79.790580,
$$

which is much greater than 1 . Thus, there exist stiff differential equations in the ODEs system for the case $\Delta x=0.3$ and DOPRI method will be applied as the estimated error of RK4. 
Table 1: Eigenvalues of Matrix $A$

\begin{tabular}{|l|l|l|l|}
\hline$\lambda_{1}=-0.566139$ & $\lambda_{2}=-1.338655$ & $\lambda_{3}=-2.228077$ & $\lambda_{4}=-3.604531$ \\
\hline$\lambda_{5}=-5.444821$ & $\lambda_{6}=-7.713231$ & $\lambda_{7}=-10.359428$ & $\lambda_{8}=-13.321281$ \\
\hline$\lambda_{9}=-16.527560$ & $\lambda_{10}=-19.900307$ & $\lambda_{11}=-23.357082$ & $\lambda_{12}=-26.813151$ \\
\hline$\lambda_{13}=-30.183668$ & $\lambda_{14}=-45.172559$ & $\lambda_{15}=-44.363593$ & $\lambda_{16}=-43.037456$ \\
\hline$\lambda_{17}=-41.226781$ & $\lambda_{18}=-38.976122$ & $\lambda_{19}=-36.340857$ & $\lambda_{20}=-33.385813$ \\
\hline
\end{tabular}

\subsection{Validation of Numerical Solutions}

To validate the numerical solutions produced using FDM, RK4 and DOPRI method, an ODE solver in MATLAB programming, ode15s is chosen for the validation. ode15s is a variable-step solver for stiff problems and it is based on the numerical differentiation formulas. Assume $\Delta x=0.3$ and $\Delta t=0.04$ whereas the tolerance value for DOPRI method and ode15s is fixed to be $\varepsilon=0.000001$. The relative errors of FDM, RK4 and DOPRI method at $b(x, 1)$ based on ode15s are stated in Table 2.

Based on the results in Table 2, the relative errors for FDM, RK4 and DOPRI method based on ode15s are small and bounded. This means that the numerical methods used are suitable to solve the disinfectant solution model. Furthermore, the relative errors produced by DOPRI method are very small and more consistent compared to RK4 when solving the model.This is because DOPRI method will automatically adjust itself to the suitable step size, $\Delta t$ in order to achieve the predetermined accuracy more efficiently.

\subsection{Accuracy of FDM and RK4}

To identify the accuracy of FDM and RK4 when solving the disinfectant solution model, a new model that resembles the behaviour of thedisinfectant solution modelis formed as shown by the following:

Model D

$$
\frac{\partial b}{\partial t}=\frac{\partial^{2} b}{\partial x^{2}}-b-e^{-x} \cdot e^{-t}, \quad 0<x<6, \quad 0<t \leq 1
$$

Initial condition:

$$
b(x, 0)=e^{-x}, \quad 0 \leq x \leq 6
$$

Boundary conditions:

$$
\begin{aligned}
& \left.\frac{\partial b}{\partial t}\right|_{x=0}=\left.\frac{\partial b}{\partial x}\right|_{x=0}, \quad 0<t \leq 1 \\
& b(6, t)=e^{-6} \cdot e^{-t}, \quad 0<t \leq 1
\end{aligned}
$$

Model D is designed for validationvia error analysis because it has an exact solution which is $b(x, t)=e^{-x} \cdot e^{-t}$. By fixing $\Delta x=0.3$ and $\Delta t=0.04$, the results executed by MATLAB programming for the relative errors of FDM and RK4 based on the exact solutions of Model D at $b(x, 1)$ are stated in Table 3. 
Table 2: Relative errors of FDM, RK4 and DOPRI method based on ode15s

\begin{tabular}{|c|c|c|c|}
\hline \multirow{2}{*}{$\boldsymbol{x}$} & \multicolumn{3}{|c|}{ Relative Error } \\
\hline & FDM & RK4 & DOPRI \\
\hline 0 & 0.003670 & 0 & 0 \\
\hline 0.3 & 0.006520 & 0 & 0 \\
\hline 0.6 & 0.010014 & 0 & 0 \\
\hline 0.9 & 0.014845 & 0 & 0 \\
\hline 1.2 & 0.017940 & 0 & 0 \\
\hline 1.5 & 0.021965 & 0 & 0.000001 \\
\hline 1.8 & 0.021301 & 0 & 0.000001 \\
\hline 2.1 & 0.021936 & 0 & 0 \\
\hline 2.4 & 0.015407 & 0 & 0.000001 \\
\hline 2.7 & 0.011888 & 0 & 0.000001 \\
\hline 3.0 & 0.000684 & 0 & 0.000002 \\
\hline 3.3 & 0.007104 & 0.000002 & 0.000001 \\
\hline 3.6 & 0.024120 & 0.000007 & 0 \\
\hline 3.9 & 0.030213 & 0.000016 & 0.000002 \\
\hline 4.2 & 0.048353 & 0.000034 & 0.000003 \\
\hline 4.5 & 0.049041 & 0.000069 & 0.000004 \\
\hline 4.8 & 0.063903 & 0.000128 & 0.000002 \\
\hline 5.1 & 0.054372 & 0.000190 & 0.000002 \\
\hline 5.4 & 0.064428 & 0.000121 & 0.000006 \\
\hline 5.7 & 0.047678 & 0.001435 & 0.000009 \\
\hline 6.0 & - & - & - \\
\hline
\end{tabular}


Table 3: Relative Errors of FDM and RK4 for Model D

\begin{tabular}{|c|c|c|}
\hline \multirow{2}{*}{$\boldsymbol{x}$} & \multicolumn{2}{|c|}{ Relative Error } \\
\hline & FDM & RK4 \\
\hline 0 & 0.032835 & 0.009194 \\
\hline 0.3 & 0.033129 & 0.007435 \\
\hline 0.6 & 0.032969 & 0.005441 \\
\hline 0.9 & 0.032393 & 0.003283 \\
\hline 1.2 & 0.031472 & 0.001046 \\
\hline 1.5 & 0.030297 & 0.001181 \\
\hline 1.8 & 0.028975 & 0.003310 \\
\hline 2.1 & 0.027610 & 0.005269 \\
\hline 2.4 & 0.026298 & 0.007003 \\
\hline 2.7 & 0.025110 & 0.008484 \\
\hline 3.0 & 0.024098 & 0.009710 \\
\hline 3.3 & 0.023274 & 0.010702 \\
\hline 3.6 & 0.022634 & 0.011503 \\
\hline 3.9 & 0.022133 & 0.012180 \\
\hline 4.2 & 0.021706 & 0.012819 \\
\hline 4.5 & 0.021230 & 0.013532 \\
\hline 4.8 & 0.020499 & 0.014423 \\
\hline 5.1 & 0.019129 & 0.015739 \\
\hline 5.4 & 0.016395 & 0.017008 \\
\hline 5.7 & 0.010903 & 0.020477 \\
\hline 6.0 & 0 & 0 \\
\hline
\end{tabular}


According to Table 3, the maximum relative errors for FDM and RK4 are 0.033129 and 0.020477, respectively. RK4 has a smaller relative error compared to FDM because the truncation error produced by RK4 is $O\left[(\Delta t)^{5},(\Delta x)^{2}\right]$ which is much smaller than that of FDM with $O\left[\Delta t,(\Delta x)^{2}\right]$. Hence, RK4 generates better accuracy of solutions for Model D compared to FDM. Since Model D follows the behaviour of the disinfectant solution model, so conclusion can be made that the numerical solutions obtained using RK4 has a higher accuracy to approximate the exact solutions for the disinfectant solution model compared to FDM.

\subsection{Numerical Simulation of Disinfectant Solution Model}

To find out the effect of different thickness of disinfectant solution on bacteria reduction, the values of $h$ are divided into $h_{1}=0.0005 \mathrm{~m}, h_{2}=0.0020 \mathrm{~m}$ and $h_{3}=0.0050 \mathrm{~m}$ to represent the thin, moderate and thick regions that applied for surface decontamination. The other parameter values as well as the range of $x$ and $t$ variables are based on the assumptions stated in Section 3.1. The spatial and time step sizes are set to be $\Delta x=0.3$ and $\Delta t=0.04$, respectively. By utilizing FDM and RK4, the numerical solutions for the dimensionless bacteria concentration at the surface area, $b(0, t)$ are separately recorded in Table 4 and Table 5 whereas the graphs are displayed in Figure 5 and Figure 6, respectively.

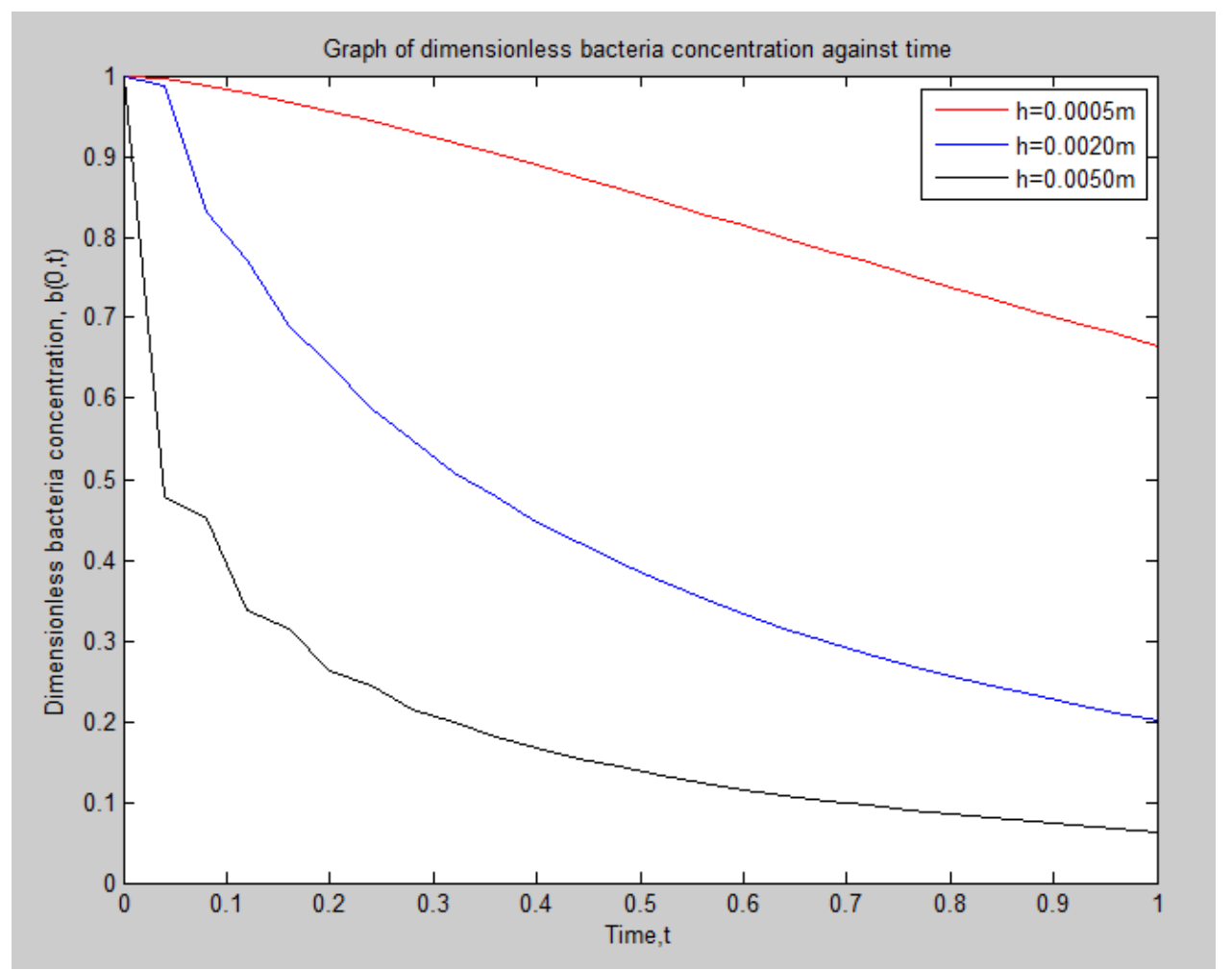

Figure 5: Dimensionless Bacteria Concentration, $b(0, t)$ against Time for Different Values of $h$ using FDM

Figure 5 and Figure 6 show that the dimensionless bacteria concentration is decreasing as the value of $h$ increases. From the graphs, $h_{3}=0.0050 \mathrm{~m}$ has the smallest amount of dimensionless bacteria concentration compared to $h_{1}=0.0005 \mathrm{~m}$ and $h_{2}=0.0020 \mathrm{~m}$. This is 
Table 4: Dimensionless Bacteria Concentration, $b(0, t)$ for Different Values of $h$ using FDM

\begin{tabular}{|c|c|c|c|}
\hline \multirow{2}{*}{$t$} & \multicolumn{3}{|c|}{ Dimensionless Bacteria Concentration, $b(0, t)$} \\
\hline & $h_{1}=0.0005 \mathrm{~m}$ & $h_{2}=0.0020 \mathrm{~m}$ & $h_{3}=0.0050 \mathrm{~m}$ \\
\hline 0 & 1.000000 & 1.000000 & 1.000000 \\
\hline 0.04 & 0.995455 & 0.986441 & 0.478152 \\
\hline 0.08 & 0.987349 & 0.831179 & 0.450575 \\
\hline 0.12 & 0.977811 & 0.772129 & 0.337349 \\
\hline 0.16 & 0.967030 & 0.689644 & 0.313817 \\
\hline 0.20 & 0.955458 & 0.641591 & 0.262968 \\
\hline 0.24 & 0.943211 & 0.587274 & 0.244139 \\
\hline 0.28 & 0.930486 & 0.548856 & 0.214708 \\
\hline 0.32 & 0.917018 & 0.509054 & 0.199601 \\
\hline 0.36 & 0.903293 & 0.477743 & 0.180072 \\
\hline 0.40 & 0.888929 & 0.446694 & 0.167801 \\
\hline 0.44 & 0.874450 & 0.420671 & 0.153693 \\
\hline 0.48 & 0.859513 & 0.395462 & 0.143591 \\
\hline 0.52 & 0.844556 & 0.373476 & 0.132804 \\
\hline 0.56 & 0.829321 & 0.352444 & 0.124386 \\
\hline 0.60 & 0.814134 & 0.333621 & 0.115808 \\
\hline 0.64 & 0.798817 & 0.315733 & 0.108714 \\
\hline 0.68 & 0.783600 & 0.299443 & 0.101698 \\
\hline 0.72 & 0.768366 & 0.284012 & 0.095664 \\
\hline 0.76 & 0.753272 & 0.269789 & 0.089806 \\
\hline 0.80 & 0.738243 & 0.256332 & 0.084630 \\
\hline 0.84 & 0.723386 & 0.243824 & 0.079664 \\
\hline 0.88 & 0.708652 & 0.231991 & 0.075191 \\
\hline 0.92 & 0.694113 & 0.220924 & 0.070930 \\
\hline 0.96 & 0.679737 & 0.210449 & 0.067041 \\
\hline 1.00 & 0.665573 & 0.200606 & 0.063352 \\
\hline
\end{tabular}


Table 5: Dimensionless Bacteria Concentration, $b(0, t)$ for Different Values of $h$ using RK4

\begin{tabular}{|c|c|c|c|}
\hline \multirow{2}{*}{$\boldsymbol{t}$} & \multicolumn{3}{|c|}{ Dimensionless Bacteria Concentration, $\boldsymbol{b}(\mathbf{0 ,} \boldsymbol{t})$} \\
\cline { 2 - 4 } & $\boldsymbol{h}_{\mathbf{1}}=\mathbf{0 . 0 0 0 5} \mathbf{~}$ & $\boldsymbol{h}_{\mathbf{2}}=\mathbf{0 . 0 0 2 0} \mathbf{~}$ & $\boldsymbol{h}_{\mathbf{3}}=\mathbf{0 . 0 0 5 0} \mathbf{~}$ \\
\hline 0 & 1.000000 & 1.000000 & 1.000000 \\
\hline 0.04 & 0.993948 & 0.940344 & 0.660103 \\
\hline 0.08 & 0.985732 & 0.859717 & 0.493092 \\
\hline 0.12 & 0.976053 & 0.783560 & 0.397843 \\
\hline 0.16 & 0.965302 & 0.716867 & 0.336209 \\
\hline 0.20 & 0.953715 & 0.659290 & 0.292380 \\
\hline 0.24 & 0.941442 & 0.609391 & 0.259077 \\
\hline 0.28 & 0.928593 & 0.565754 & 0.232578 \\
\hline 0.32 & 0.915255 & 0.527219 & 0.210789 \\
\hline 0.36 & 0.901502 & 0.492880 & 0.192434 \\
\hline 0.40 & 0.887405 & 0.462032 & 0.176685 \\
\hline 0.44 & 0.873028 & 0.434126 & 0.162977 \\
\hline 0.48 & 0.858432 & 0.408729 & 0.150905 \\
\hline 0.52 & 0.843672 & 0.385495 & 0.140175 \\
\hline 0.56 & 0.828800 & 0.364141 & 0.130561 \\
\hline 0.60 & 0.813862 & 0.344439 & 0.121890 \\
\hline 0.64 & 0.798900 & 0.326197 & 0.114026 \\
\hline 0.68 & 0.783952 & 0.309256 & 0.106860 \\
\hline 0.72 & 0.769050 & 0.293478 & 0.100301 \\
\hline 0.76 & 0.754223 & 0.278750 & 0.094277 \\
\hline 0.80 & 0.739496 & 0.264972 & 0.088726 \\
\hline 0.84 & 0.724891 & 0.252056 & 0.083596 \\
\hline 0.88 & 0.710426 & 0.239929 & 0.078842 \\
\hline 0.92 & 0.696118 & 0.228523 & 0.074429 \\
\hline 0.96 & 0.681981 & 0.217781 & 0.070322 \\
\hline 1.00 & 0.668025 & 0.207650 & 0.066493 \\
\hline & & & \\
\hline & & & $b$ \\
\hline
\end{tabular}




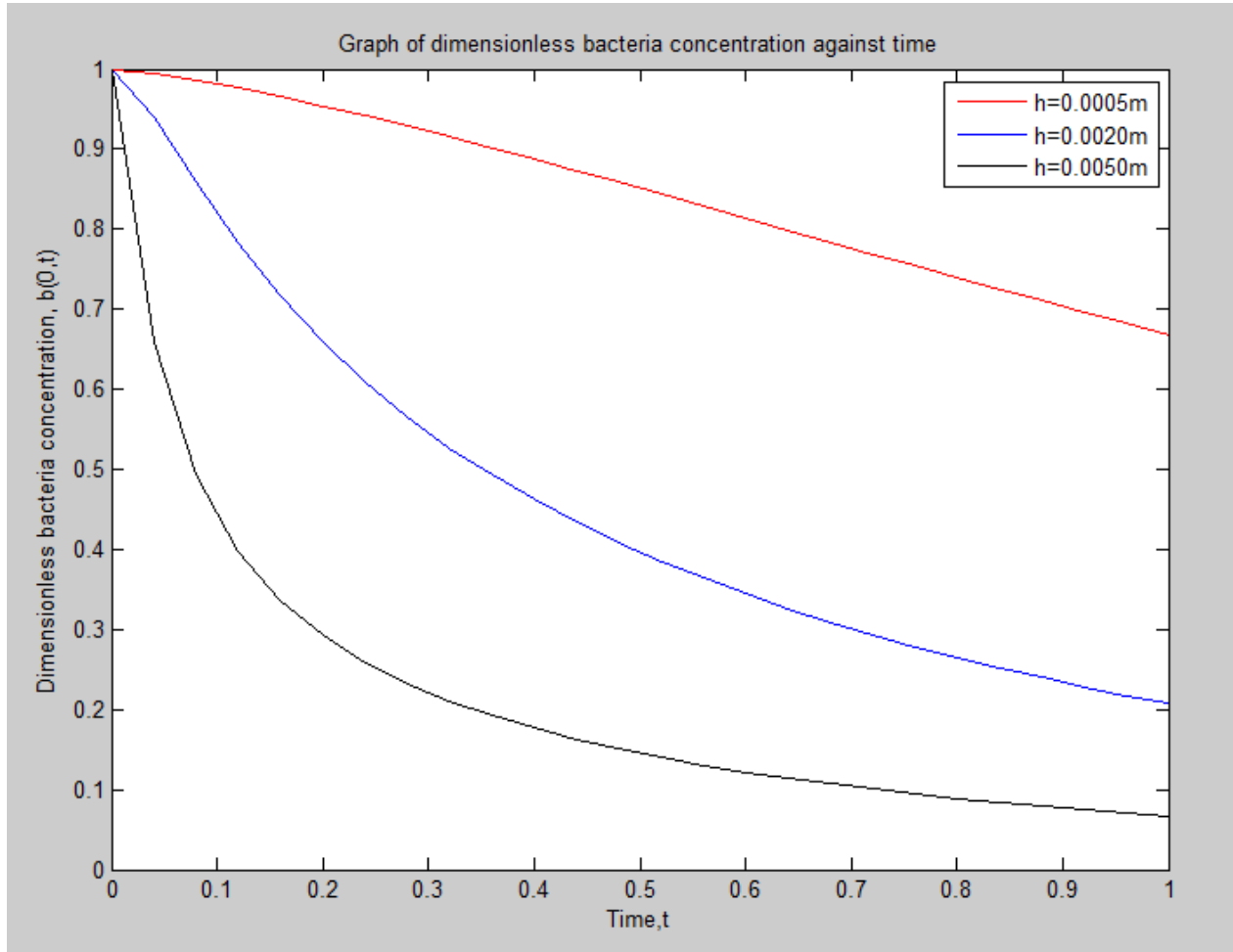

Figure 6: Dimensionless Bacteria Concentration, $b(0, t)$ against Time for Different Values of $h$ using RK4

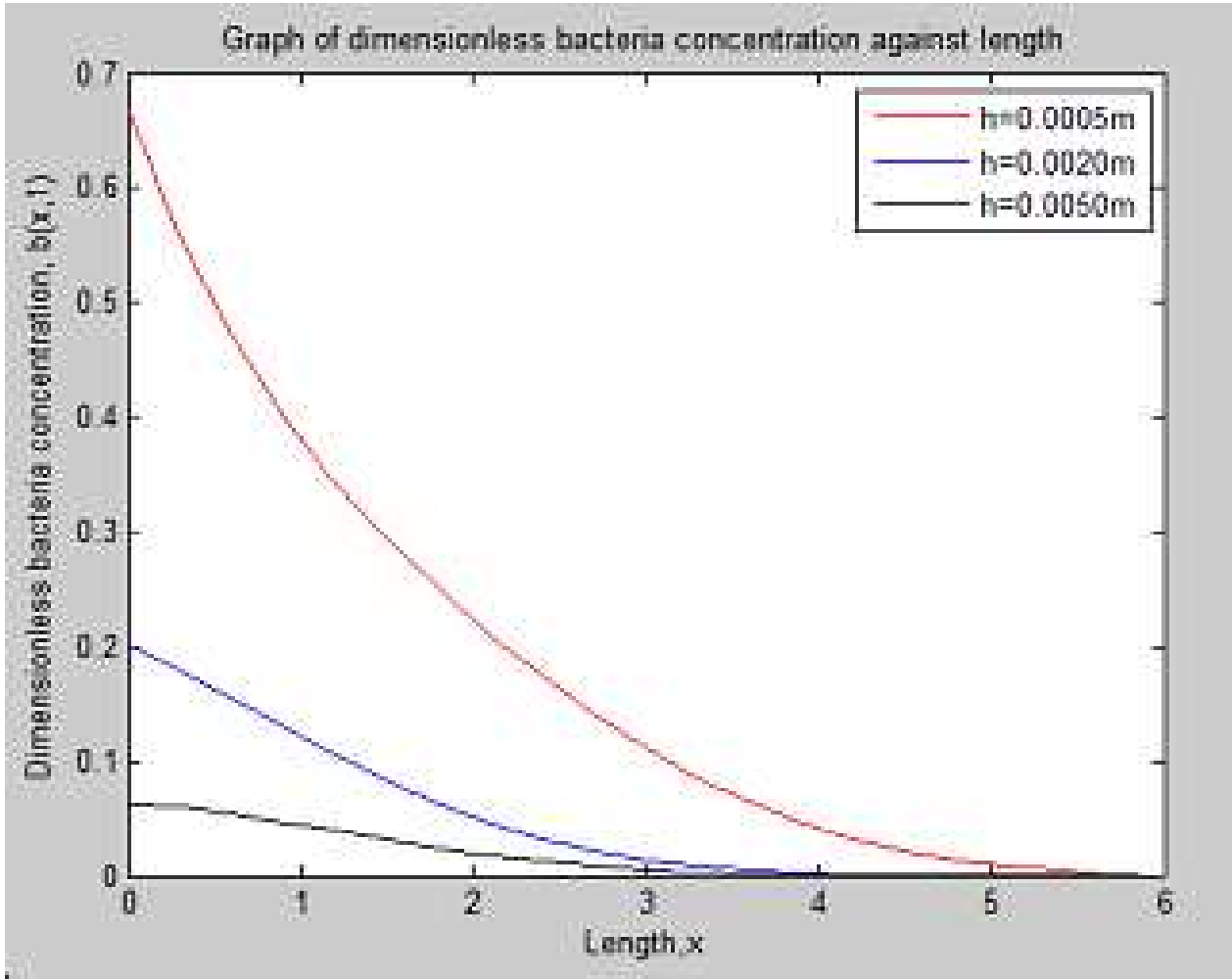

Figure 7: Dimensionless Bacteria Concentration, $b(x, 1)$ against $x$ for Different Values of $h$ using FDM 


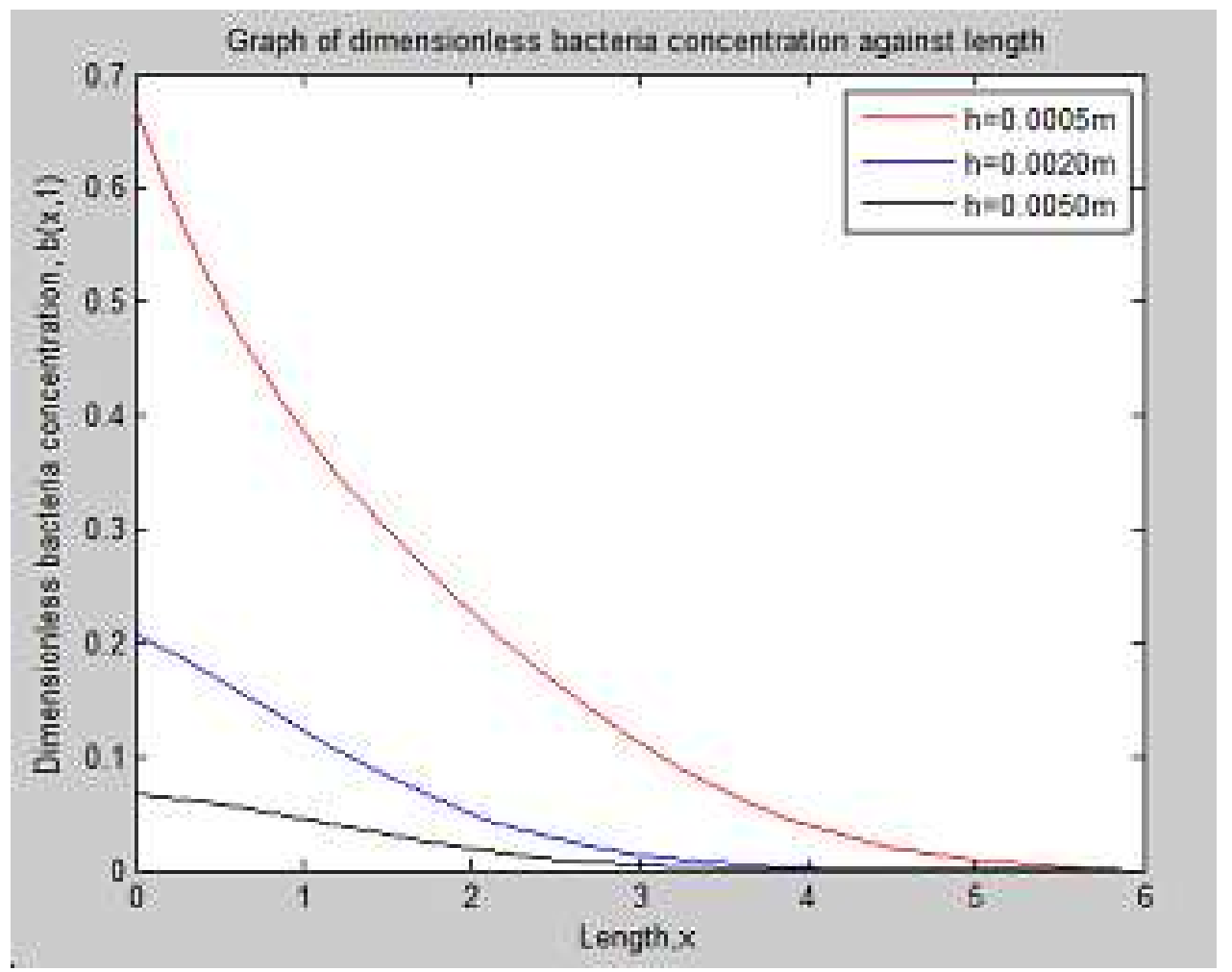

Figure 8: Dimensionless Bacteria Concentration, $b(x, 1)$ against $x$ for Different Values of $h$ using RK4

reasonable because the increasing of $h$ will provide larger space for the bacteria at the surface area to diffuse into the disinfectant solution and then being eliminated by the disinfectant killing effect. Therefore, thick disinfectant solution is able to reduce the dimensionless bacteria concentration more effectively. Figure 7 and 8 indicate that the disinfectant solution obey the boundary condition where solution tend to zero when $x$ tend to domain length.

\section{Conclusion}

In a nutshell, RK4 has a larger stability region and higher accuracy of solutions compared to FDM when solving the disinfectant solution model. This implies that RK4 is able to generate stable numerical solutions that can approximate the exact solutions more accurately than FDM. In addition, different thickness of disinfectant solution did affect the amount of dimensionless bacteria concentration found at the decontaminated surface. The thicker the disinfectant solution applied for surface decontamination, the greater the killing effect on bacteria reduction. This means that thick disinfectant solution promotes the diffusion process of bacteria into disinfectant solution region. Consequently, more bacteria are killed by the antibacterial property of disinfectant. 


\section{Acknowledgement}

We would like to express our deepest gratitude to John R. Ockendon, F. R. S., Colin P. Please and Cameron Hall from the Oxford Centre for Industrial and Applied Mathematics (OCIAM), University of Oxford, for contributing ideas in constructing mathematical modelling for the surface decontamination by disinfectant solution [5]. Without this model, this research would not be accomplished successfully. This work was supported in part by the Malaysian Ministry of Education through the Research Management Centre (RMC), Universiti Teknologi Malaysia (Ref. No. Q.J130000.2426.03G51). This support is gratefully acknowledged.

\section{References}

[1] Otto, C. C. and Haydel, S. E. Exchangeable ions are responsible for the in vitro antibacterial properties of natural clay mixtures. PLOS ONE. 2013. 8(5): e64068.

[2] Williams, L. B. and Haydel, S. E. Evaluation of the medicinal use of clay minerals as antibacterial agents. International Geology Review. 2010. 52(7-8): 745-770.

[3] Williams, L. B., Haydel, S. E., Giese Jr., R. F. and Eberl, D. D. Chemical and mineralogical characteristics of French green clays used for healing. Clays and Clay Minerals. 2008. 56(4): 437.

[4] Williams, L. B., Metge, D. W., Eberl, D. D., Harvey, R. W., Turner, A. G., Prapaipong, P. and Poret-Peterson, A. T. What makes a natural clay antibacterial?. Environmental Science \& Technology. 2011. 45(8): 3768-3773.

[5] Ockendon, J. R., F. R. S., Please, C. P., Hall, C., Murid, A. H. M., Yeak, S. H. and Hamzah, A. S. Report on Mathematical Modelling of Surface Decontamination by Clay Solution. Unpublished report. 2016.

[6] Chen, J. S., Liang, C. P., Gau, H. S. and Liu, C. W. Mathematical model for formation decontamination by pumping with well bore mixing. Applied Mathematical Modelling. 2006. 30(5): 446-457.

[7] Alavani, C., Glowinski, R., Gomez, S., Ivorra, B., Joshi, P. and Ramos, A. M. Modelling and simulation of a polluted water pumping process. Mathematical and Computer Modelling. 2010. 51(5): 461-472.

[8] Miao, T. and Pan, T. A multiphysics model for evaluating electrokinetic remediation of nuclear waste-contaminated soils. Water, Air, $\&$ Soil Pollution. 2015. 226(3): 1-12.

[9] Schiesser, W. E. and Griffiths, G. W. A Compendium of Partial Differential Equation Models: Method of Lines Analysis with Matlab. USA: Cambridge University Press. 2009.

[10] Islam, M. A. Accurate solutions of initial value problems for ordinary differential equations with the fourth order Runge Kutta method. Journal of Mathematics Research. 2015. 7(3): 41-45.

[11] Lambert, J. D. Computational Methods in Ordinary Differential Equations. New York: Wiley. 1974. 